OPEN ACCESS

Edited by:

Vicent Arbona,

Jaume I University, Spain

Reviewed by:

Umesh K. Reddy,

West Virginia State University,

United States

Huize Chen,

Institute for Basic Science,

South Korea

${ }^{*}$ Correspondence:

Fei Xiong

feixiong@yzu.edu.cn

Specialty section:

This article was submitted to

Plant Abiotic Stress,

a section of the journal

Frontiers in Plant Science

Received: 01 June 2017 Accepted: 19 September 2017

Published: 04 October 2017

Citation:

Chen X-y, Yang Y, Ran L-p, Dong Z-d, Zhang E-j, Yu X-r and Xiong F (2017) Novel Insights into miRNA Regulation of Storage Protein Biosynthesis during Wheat Caryopsis Development under doi: 10.3389/fp/s.2017.01707

\section{Novel Insights into miRNA Regulation of Storage Protein Biosynthesis during Wheat Caryopsis Development under Drought Stress}

\author{
Xin-yu Chen, Yang Yang, Li-ping Ran, Zhao-di Dong, Er-jin Zhang, Xu-run Yu and \\ Fei Xiong *
}

Jiangsu Key Laboratory of Crop Genetics and Physiology, Co-Innovation Center for Modern Production Technology of Grain Crops, Joint International Research Laboratory of Agriculture and Agri-Product Safety, Yangzhou University, Yangzhou, China

Drought stress is a significant abiotic stress factor that affects wheat yield and quality. MicroRNA (miRNA) plays an important role in regulating caryopsis development in response to drought stress. However, little is known about the expression characteristics of miRNAs and how they regulate protein accumulation in wheat caryopsis under drought stress. To address this, two small RNA libraries of wheat caryopsis under control and drought stress conditions were constructed and sequenced. A total of 125 miRNAs were identified in the two samples, of which 110 were known and 15 were novel. A total of 1,981 miRNA target genes were predicted and functional annotations were obtained from various databases for 1,641 of them. Four miRNAs were identified as differential expression under drought stress, and the expression patterns of three of them were consistent with results obtained by reverse transcription polymerase chain reaction (RT-PCR) and reverse transcription quantitative polymerase chain reaction (RT-qPCR). Moreover, three miRNA-target pairs showed negative regulation tendency, as revealed by RT-qPCR. Functional enrichment and pathway analysis revealed that four pathways might be involved in storage protein biosynthesis. Furthermore, drought stress significantly increased the accumulation of protein bodies and protein content in wheat endosperm. In summary, our findings suggest that drought stress may enhance storage protein by regulating the expression of miRNAs and their target genes.

Keywords: wheat, drought stress, microRNA, storage protein, protein body

\section{INTRODUCTION}

Drought stress is a major abiotic stress factor that poses a significant challenge to global crop production. Long term or periodic droughts greatly affect crop growth and yield formation. Therefore, investigating drought resistance of crops is critical for achieving long-term productivity gains (Lawlor, 2013). Wheat is an important cereal crop that is widely cultivated around the world and is sensitive to drought stress during vegetative and reproductive growth (Liu et al., 2005). In response to drought stress, wheat exhibits systematic adaptability in morphological structure, physiology, biochemistry, and gene regulation (Chaves et al., 2003). When subjected to drought stress, expression of relevant stress-resistant genes is induced and regulated by molecule-mediated sensory and conduction signaling systems. These include effector and regulatory molecules that 
are directly involved in biochemical responses and expression products of non-encoding genes in wheat (Shinozaki and Yamaguchi-Shinozaki, 2007).

MicroRNA (miRNA), an endogenous non-coding singlestranded RNA, is highly conserved and widely present in eukaryotes. Mature miRNAs, which are usually 21-24 nucleotides in length, are a kind of important regulatory factor of gene expression in eukaryotes (Ambros, 2004). Transcription of miRNA-encoding genes is catalyzed by RNA polymerase II, and the transcripts are spliced into mature miRNA via dicer-like enzymes and several protein complexes (Bartel, 2004). Based on complementarities between miRNA and target genes, miRNAs regulate target gene expression via two mechanisms, namely, cleavage-induced targeted mRNA degradation and translational repression (Mallory and Bouché, 2008). In plants, most miRNAs display perfect or near perfect complementarity with their mRNA targets. Thus, plant miRNAs regulate the expression of target genes primarily through specific mRNA cleavage (Rhoades et al., 2002; Chen, 2005).

miRNA plays an essential role in plant development and stress adaptation. By performing high-throughput sequencing of all small RNAs in samples, miRNA expression profiles can be obtained at the whole genome level without any sequence information. This can then be used for discovery of novel miRNAs, prediction of target genes, and differential expression analysis, which may greatly promote the discovery and functional study of plant miRNAs (Buermans et al., 2010). Recent studies have shown that miRNAs are involved in regulating responses to various biotic and abiotic stresses in plants (Covarrubias and Reyes, 2009; Jagadeeswaran et al., 2009). In rice, miR169g expression is significantly up-regulated under drought stress, whereas miR393 only exhibits transient expression (Zhao et al., 2007). High-throughput sequencing was used to study the small RNAs in potatoes under drought stress. Numerous conserved and specific miRNAs were identified and a negative correlation between drought-related miRNAs and target genes was found (Zhang et al., 2014). It is widely believed that stress conditions change the expression of miRNAs, which in turn leads to changes in expression of miRNA targets (Reyes and Chua, 2007). Thus, functional analysis of targets can be helpful for understanding the mechanism underlying the regulatory role of miRNA in stress responses. Song et al. (2012) identified the target of miR394 which encoded an F-box protein (At1g27340) involved in the regulation of leaf curling-related morphology in Arabidopsis. Additionally, miRNA can affect plant growth under stress conditions by regulating signaling pathways. MiR393 was found to target transport inhibitor response 1 (TIR1), which is known as an auxin receptor and positive regulator of auxin signaling (Windels and Vazquez, 2011). To validate these target genes of miRNA, degradome sequencing can be used (Zhou M. et al., 2010). Li et al. (2015) investigated development-related miRNAs and their validated targets during wheat grain development by small RNA sequencing combined with degradome sequencing.

Wheat caryopsis is an important organ for nutrient storage, and its development determines the yield and quality of wheat. Drea et al. (2005) carried out a systematic analysis of gene expression during caryopsis development and revealed novel distinct spatial expression patterns that either indicated the ontogeny of the developing caryopsis or reflected specialized cellular function. Protein, which is one of the main storage substances in wheat endosperm, accounts for $10-15 \%$ of the dry weight of mature wheat seeds (Tasleem-Tahir et al., 2012). In wheat, as in other cereal crops, storage proteins, including gliadin, glutenin, and globulin, accumulate in an endosperm organelle with the development of caryopsis, protein body (PB), which may be formed in the rough endoplasmic reticulum or protein storage vacuoles (Chen et al., 2014). Protein content and $\mathrm{PB}$ amount directly affect the processing and nutritional quality of wheat flour (Kindred et al., 2008). The biosynthesis of storage protein is generally considered to be regulated at the transcriptional level (Verdier and Thompson, 2008). Previous studies have indicated that some genes are not directly involved in storage protein gene expression but may influence storage protein biosynthesis, such as those encoding glutamate synthetase and glutamine synthase (Habash et al., 2009). However, the expression characteristics and regulation mechanism of miRNAs during wheat caryopsis development, particularly the protein biosynthesis and $\mathrm{PB}$ development under drought stress, remain to be unknown. To investigate this issue, high-throughput sequencing of miRNA was conducted, and the differentially expressed miRNAs in wheat caryopsis under drought stress were identified. In addition, the miRNA target genes were predicted and functional analysis of target genes was performed to investigate the possible mechanism underlying miRNA regulation of storage protein biosynthesis in wheat endosperm when subjected to drought stress. Our study may inform future studies on mechanisms underlying wheat drought resistance and may provide a theoretical basis for genetic breeding of drought-tolerant wheat cultivars.

\section{MATERIALS AND METHODS}

\section{Plant Materials and Treatment}

The wheat cultivar selected in this study was Yangmai 16 provided by the Agricultural College of Yangzhou University, Jiangsu Province, China. Seeds were sown in plastic pots (20 seeds per pot) that were placed in rainproof shelters in the experimental field of Key Laboratory of Crop Genetics and Physiology in Yangzhou University from 2015 to 2016 . The soil was sandy loam and contained organic substances (2.45\%), nitrogen $(106 \mathrm{mg} / \mathrm{kg})$, phosphorus $(33.8 \mathrm{mg} / \mathrm{kg})$, and available potassium $(66.4 \mathrm{mg} / \mathrm{kg})$. Seedlings were thinned to eight plants per pot 2 weeks after sowing. A minupressure soil hygrometer (SP-11, Institute of Soil Science in Nanjing, China) was used to detect the water potential at $15 \mathrm{~cm}$ in the soil. Watering was strictly controlled from plant re-greening (the stage before erecting and after wintering) to the caryopsis mature stage, maintaining water potential at -20 and $-60 \mathrm{kPa}$. These values corresponded to the optimum level of normal water supply condition and drought stress, respectively (Figure 1). All other steps for management of the control and treatment groups throughout the growth cycle were the same. Each treatment group consisted of 30 pots. During wheat flowering, two individual florets at the base of the central ears were marked with a marker pen to label the anthesis date. 

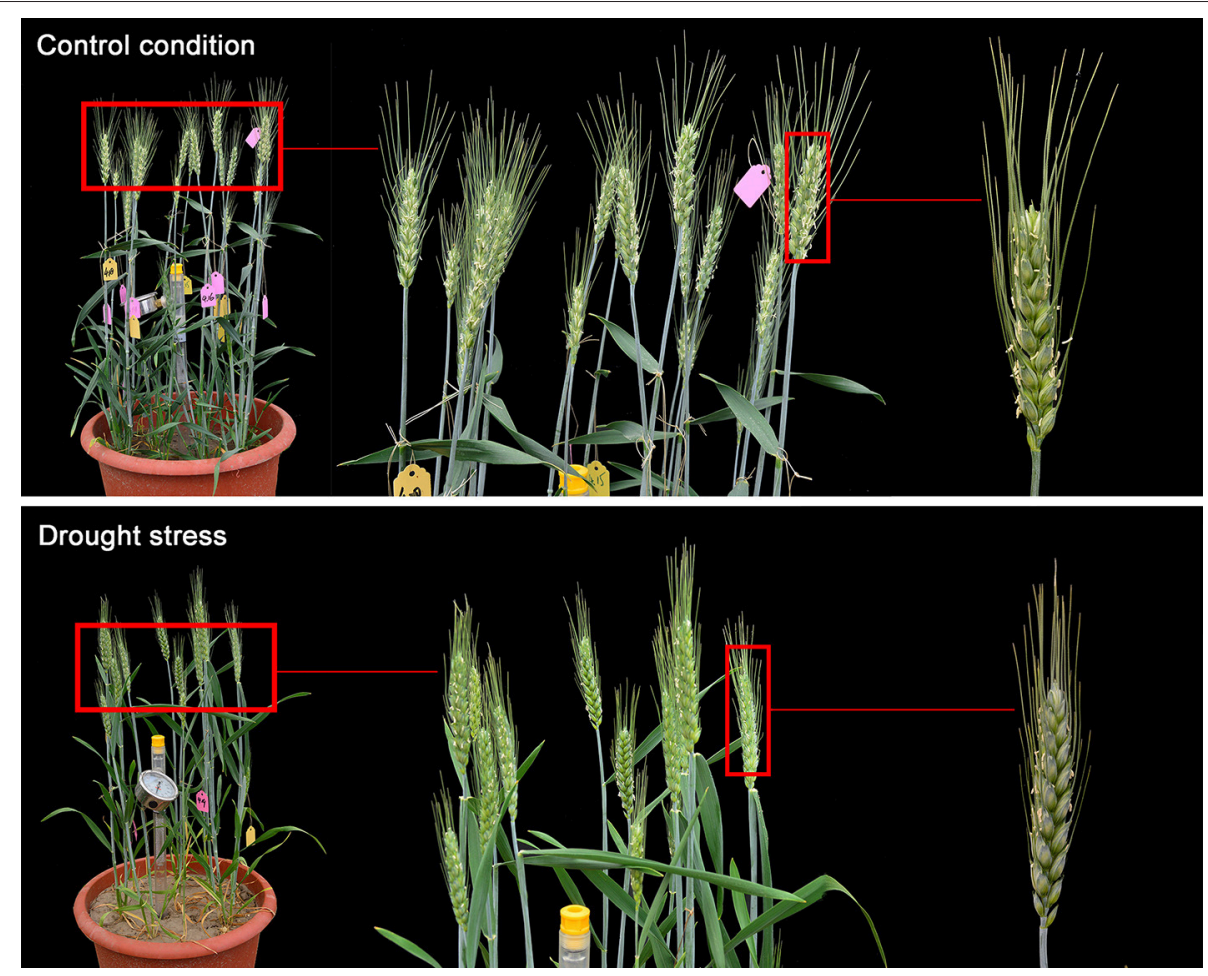

FIGURE 1 | Wheat plant under control and drought stress conditions. Wheat was planted in plastic pots maintaining soil water potential at -20 and -60 kPa, which corresponded to control and drought stress conditions, respectively. Under control condition, wheat plant grew well while wheat leaves grew yellow under drought stress. Meanwhile, the wheat ears under drought stress were smaller and the grains were not as full as those under control.

\section{RNA Extraction and Sequencing}

Wheat caryopses under control and drought stress were collected 15 days after anthesis (DAA) and frozen immediately in liquid nitrogen. The collected samples were made up of caryopses from the middle of six different wheat ears and each two ears were from three different plastic pots. Frozen tissues were pulverized in liquid nitrogen using a mortar and pestle. Total RNAs were extracted using Trizol Reagent (Shanghai Sangon Biotech Co. Ltd., China) following the manufacturer's instructions. The purity, concentration, and integrity of RNA samples were assayed using Nanodrop, Qubit2.0, and Agilent 2,100 bioanalyzer, respectively. Two small RNA libraries were constructed using the small RNA Sample Pre Kit. Briefly, the extracted total RNA was ligated to $5^{\prime}$ and $3^{\prime}$ adapters using T4 RNA Ligase 1 and T4 RNA Ligase 2, respectively. Subsequently, the cDNA was synthesized by reverse transcription and amplified using polymerase chain reaction (PCR). Then the products were purified via PAGE to attain small RNA libraries. Finally, the quality control of libraries was performed using Qubit 2.0, Agilent 2,100 bioanalyzer, and quantitative PCR. The resulting RNA was sequenced by Nanjing Genepioneer Biotechnologies using the Illumina HiSeq2500 high-throughput sequencing platform.

\section{Basic Analysis of Sequencing Data}

The raw image data files from sequencing were converted to raw data via base calling, and clean reads were obtained by filtering out the $5^{\prime}$ terminal contamination or low quality sequences. The effective reads were eventually obtained after quality control of the clean reads. The effective reads were aligned to Rfam and Repbase databases using Bowtie (Langmead, 2010) software. The small RNA was annotated according to the priority order of rRNA $>$ tRNA $>$ snRNA $>$ snoRNA $>$ repeat. The unclassified ncRNA tags were aligned with the reference genome using miRDeep2 software (Friedländer et al., 2012) to obtain the position information on the reference genome. Then, sequence length distribution as well as common and specific sequence analysis were conducted.

\section{miRNA Analysis}

Sequences mapped to reference genome were used for known and novel miRNA identification using miRDeep2 software. The effective reads mapped to reference genomes were aligned against precursor and mature sequences of known wheat miRNA in miRBase. If precursor sequence, mature sequence, and hairpin structure were consistent, the effective reads were regarded as known miRNAs. Novel miRNA prediction was done using miRDeep2 software, which can identify novel miRNA by predicting hairpin structure, analyzing Dicer enzyme splice sites, and calculating free energy. Moreover, length distribution statistics and base preference analysis of identified miRNAs were performed. 
TABLE 1 | Primers for internal control genes, differentially expressed miRNAs and corresponding target genes used in RT-qPCR validation.

\begin{tabular}{llll}
\hline Type & Gene/miRNA & Forward primer $\mathbf{5}^{\prime} \rightarrow \mathbf{3}^{\prime}$ & Reverse primer $\mathbf{5}^{\prime} \rightarrow \mathbf{3}^{\prime}$ \\
\hline Target gene & Ta54825 & TGACCGTATGAGCAAGGAG & CCAGACAACTCGCAACTTAG \\
& TC436629 & CGACTGCTCTCCTACCT & GATGCCGTTGAGGACCT \\
& TC369499 & GTCGCTCCATTGCCTACTT & GACTGGCTCATTGTCC \\
& TC371915 & GGCGTGCTTCTGGGTAT & GCTTCAAATGTGGTGGG \\
& DR733425 & GCTCCACCTACATCTCG & CTCTGCTGCTTCTCAA \\
\hline miRNA & ta & cttcggggacatccgataaaattg & - \\
& tae-nsmR10 & atatAGGCGCCCTGGGGGGCCGAACGGC & - \\
& tae-nsmR5/6 & tatataCGCGAtGAtGGCCGCGCGGGCtCAC & - \\
& tae-miR9654a-3p & AAACCCGGACtGtGtCGtAtGtGC & -
\end{tabular}

Lowercase, RNA; uppercase, DNA. The reverse primers used for RT-qPCR of miRNAs are provided by the kit. Sequences are not listed in this study to preserve product confidentiality.

TABLE 2 | Statistics of small RNA classification annotation.

\begin{tabular}{|c|c|c|c|c|c|c|c|c|}
\hline Type & \multicolumn{4}{|c|}{ Control } & \multicolumn{4}{|c|}{ Drought stress } \\
\hline tRNA & 312093 & 2.01 & 31109 & 0.64 & 184818 & 1.79 & 23654 & 0.69 \\
\hline snRNA & 23444 & 0.15 & 8931 & 0.18 & 14992 & 0.14 & 6798 & 0.20 \\
\hline snoRNA & 63273 & 0.41 & 14541 & 0.30 & 38836 & 0.38 & 10872 & 0.32 \\
\hline Unmapped & 1113425 & 7.17 & 417220 & 8.62 & 566168 & 5.48 & 270962 & 7.91 \\
\hline Total & 15536948 & 100 & 4842873 & 100 & 10340923 & 100 & 3425081 & 100 \\
\hline
\end{tabular}

\section{Differential Expression Analysis of miRNA}

To identify the differentially expressed miRNAs, expression levels of miRNAs were compared between the control and drought stress samples. Based on the sequencing data, miRNA expressions in the two samples were counted and normalized to obtain transcript per million using a normalization formula described previously (Zhou L. et al., 2010). Then, the fold change and $p$ values were calculated from the normalized expressions. EdgeR was used to conduct the differential expression analysis, which ran a web tool IDEG6 to detect differentially expressed miRNAs (Romualdi et al., 2003). The differentially expressed miRNAs were screened at a standard of $|\log 2(\mathrm{FC})| \geq 1$ and $p$-value $<0.05$.

\section{Reverse Transcription PCR (RT-PCR) of Differentially Expressed miRNA}

Total RNA was extracted from wheat caryopses under control and drought conditions using Trizol reagent according to the manufacturer's instructions. RNA was reverse transcribed to cDNA using One-Step miRNA RT Kit (SinoGene, China). The reverse transcription reaction mixture contained $12 \mu \mathrm{L}$ RNA template mix, $1 \mu \mathrm{L}$ Oligo-dT adapter primer, $4 \mu \mathrm{L} 5$ $\times$ reaction buffer, $1 \mu \mathrm{L}$ M-MLV RTase and $2 \mu \mathrm{L} 10 \mathrm{mM}$ of dNTP. The reverse transcription reaction was performed at $37^{\circ} \mathrm{C}$ for $60 \mathrm{~min}$ followed by $85^{\circ} \mathrm{C}$ for $10 \mathrm{~min}$. RT-PCR was performed for 4 miRNAs using cDNA as template and U6 served as internal control to normalize the cDNA concentrations. PCR amplification was performed in a $25 \mu \mathrm{L}$ reaction volume containing $0.5 \mu \mathrm{L}$ Taq enzyme, $2.5 \mu \mathrm{L}$ cDNA template, $3 \mu \mathrm{L}$ $10 \times$ buffer (including $\mathrm{MgCl}_{2}$ ), $1 \mu \mathrm{L}$ dNTP, $1 \mu \mathrm{L}$ of each primer at the concentration of $20 \mathrm{mM}$, and $11 \mu \mathrm{L}$ nucleasefree $\mathrm{ddH}_{2} \mathrm{O}$. The condition of PRC was set as follows: $95^{\circ} \mathrm{C}$ for $3 \mathrm{~min}$, followed by $25-35$ cycles of $94^{\circ} \mathrm{C}$ for $30 \mathrm{~s}, 52^{\circ} \mathrm{C}$ for $30 \mathrm{~s}$, and $68^{\circ} \mathrm{C}$ for $30 \mathrm{~s}$, depending upon the individual miRNA. Final extension was performed at $68^{\circ} \mathrm{C}$ for $7 \mathrm{~min}$. Amplified products were separated on $1.5 \%$ agarose gel and the marker used was NormalRun ${ }^{\mathrm{TM}}$ prestained 250 bp-I DNA ladder (Generay, China).

\section{Prediction and Functional Annotation of miRNA Target Genes}

The target genes of miRNA were predicted using psRNATarget (Enright et al., 2003) according to sequence alignment between identified miRNAs and wheat genomes. Non-redundant protein sequences, Swiss-Prot, gene ontology (GO), Cluster of Orthologous Groups of proteins, Kyoto Encyclopedia of Genes and Genomes (KEGG), Eukaryotic Ortholog Groups, and Pfam database were used to annotate the function of target genes using BLAST software. GO enrichment (Consortium et al., 2000) and 
A

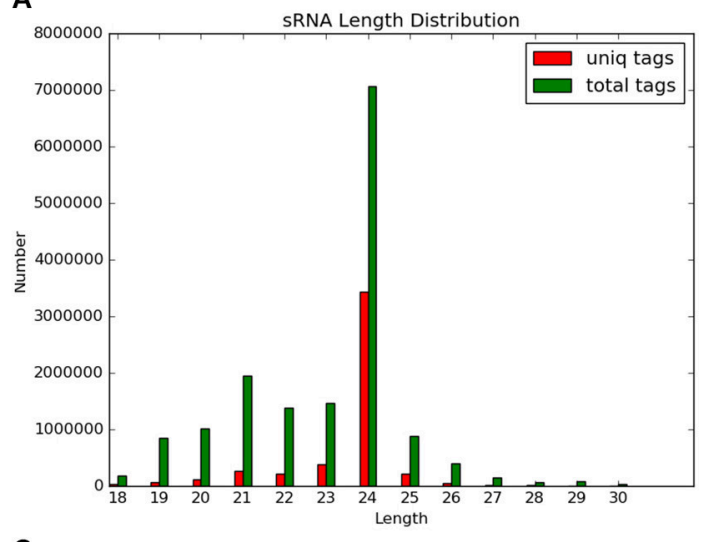

C

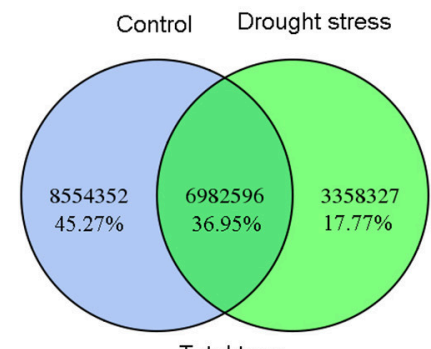

B
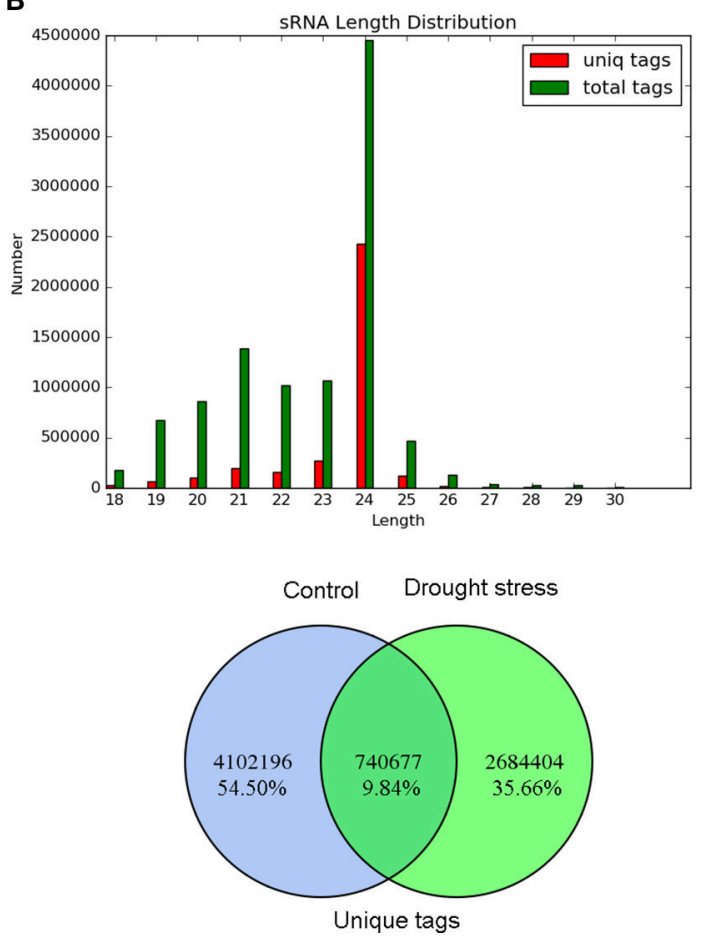

FIGURE 2 | Sequence analysis of small RNAs. (A) Length distribution of small RNAs under control. (B) Length distribution of small RNAs under drought stress. The distributions of total tags and unique tags are shown as numbers. (C) Venn diagram of common and specific sequence statistics between control and drought stress. Total tags and unique tags are shown in the left and right panels, respectively.
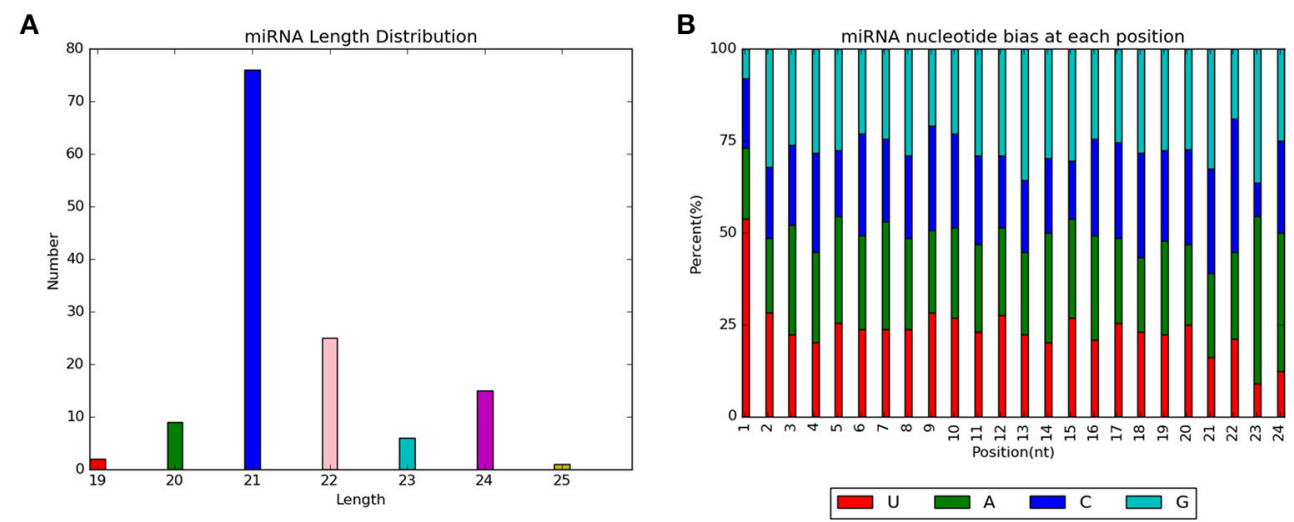

FIGURE 3 | Analysis of all identified miRNAs from control and drought stress samples. (A) Length distribution of all miRNAs. (B) Nucleotide biases at each position for all miRNAs.

KEGG pathway analysis (Kanehisa et al., 2004) were undertaken on target genes of differentially expressed miRNAs.

\section{Microstructure Observation of Wheat Caryopsis}

Wheat caryopses at 15 DAA were acquired and cut transversely into $2 \mathrm{~mm}$ slices from the middle using a razor blade. The caryopsis slices were soaked in $2.5 \%$ glutaraldehyde fixative [25\% glutaraldehyde diluted 10 times at $\mathrm{pH} 7.2$ phosphatebuffered solution (PBS)] at $4{ }^{\circ} \mathrm{C}$ for $48 \mathrm{~h}$ immediately. The fixed samples were subsequently rinsed thrice with PBS and dehydrated in a graded ethanol series [20, 40, 60, 80, 90, 95, and $100 \%$ (thrice)], followed by propylene oxide replacement. Afterward, the samples were infiltrated and embedded in low-viscosity Spurr's resin and polymerized at $70^{\circ} \mathrm{C}$ for $12 \mathrm{~h}$. The samples were cut into $1 \mu \mathrm{m}$ slices using an ultramicrotome (Ultracut R, Leica, Germany) and pasted onto glass slides. Then, the slices were stained with $0.5 \%$ methyl violet, rinsed, dried, and observed under a light microscope (DMLS, Leica, Germany). Photographs were 
TABLE 3 | Sequence information of putative novel miRNA.

\begin{tabular}{|c|c|c|c|}
\hline \multicolumn{2}{|c|}{ Conservative miRNA } & \multicolumn{2}{|c|}{ Unconservative miRNA } \\
\hline miRNA name & Mature miRNA sequence & miRNA name & Mature miRNA sequence \\
\hline tae-csmR156-1 & UGACAGAAGAGAGUGAGCAC & tae-nsmR1 & UCGGCUACUUCCUUUCCCUUGC \\
\hline tae-csmR5082-1 & CGCGAUGAUGGCCGCGCGGGCUCAC & tae-nsmR2 & UCACUGUUUGGAAAGGUAGGAC \\
\hline tae-csmR7743-1 & AUUGAACUAAGGAGGGGUGGA & tae-nsmR3 & CGCGGCUCCGUCGACUGGUGC \\
\hline tae-csmR172-1 & GCAGCACCACCAAGAUUCACA & tae-nsmR4 & GUCUCUGCCAAUUCUUCGUGU \\
\hline \multirow[t]{6}{*}{ tae-csmR2275-1 } & UUUGGUUUGAAGGGAGCUCUG & tae-nsmR5 & AAACCCGGACUGUGUCGUAUGUGC \\
\hline & & tae-nsmR6 & AAACCCGGACUGUGUCGUAUGUGC \\
\hline & & tae-nsmR7 & AGCCCGCGGCAUGGCGUCGCA \\
\hline & & tae-nsmR8 & UUAGGAACAUGUGUGCUCUCUUGU \\
\hline & & tae-nsmR9 & CAUCCCCUUCGCCGGCUGCGC \\
\hline & & tae-nsmR10 & AGGCGCCCUGGGGGGCCGAACGGC \\
\hline
\end{tabular}

captured using a camera (Truechrome II, Truechrome, China) attached to the light microscope. Each treatment selected three caryopses as repeats, each of which was from different wheat spikes.

\section{Protein Content Determination}

Wheat caryopses were collected at 15 and 45 DAA (mature stage) and dried at $42^{\circ} \mathrm{C}$ to a constant weight. The samples were then completely ground using a mortar and the powder was filtered through a 100 mesh wire screen. Up to 200 mg of the sample was mixed with the catalyst $\left(\mathrm{CuSO}_{4}+\right.$ $\mathrm{K}_{2} \mathrm{SO}_{4}$ ). The nitrogen content (\%) was determined on an automatic Kjeldahl apparatus (FOSS KjeltecTM8400, Analyzer, Unit, Hoganas) after digesting the concentrated sulfuric acid. Sample protein content (\%) was calculated by multiplying the coefficient 5.7. Three individual replicates were used for each sample.

\section{Reverse Transcription Quantitative PCR (RT-QPCR) Analysis of Differentially Expressed miRNAs and Target Genes}

Total RNA was extracted from both control and drought-treated samples using Trizol reagent according to the manufacturer's instructions. For miRNA, $5 \mu \mathrm{g}$ of total RNA was polyadenylated with a poly (A) adapter and reverse-transcribed into cDNA using One-Step miRNA RT Kit (SinoGene, China). The sequence complementary to the poly(A) adapter was used as the primer. Reverse transcription reaction was performed in $20 \mu \mathrm{L}$ reaction mixture (RNA Template Mix $12 \mu \mathrm{L}$, Oligo-dT Adapter Primer $1 \mu \mathrm{L}, 5 \times$ Reaction Buffer $4 \mu \mathrm{L}, \mathrm{M}$-MLV RTase $1 \mu \mathrm{L}, 10 \mathrm{mM}$ of dNTP $2 \mu \mathrm{L}$ ) at $37^{\circ} \mathrm{C}$ for $60 \mathrm{~min}$ followed by $85^{\circ} \mathrm{C}$ for $10 \mathrm{~min}$. For target gene, $5 \mu \mathrm{g}$ of total RNA was reverse-transcribed into cDNA using PrimeScript ${ }^{\mathrm{TM}}$ RT reagent Kit (TaKaRa, Japan). Reverse transcription reaction was performed in a $20 \mu \mathrm{L}$ reaction mixture containing total RNA mix, $4 \mu \mathrm{L} 5 \times$ PrimeScript buffer (including $\mathrm{dNTP}$ mixture and $\mathrm{Mg}^{2+}$ ), $1 \mu \mathrm{L}$ PrimeScript $\mathrm{RT}$ enzyme mix I, $1 \mu \mathrm{L}$ oligo dT primer, $1 \mu \mathrm{L}$ random 6 mers, and RNase-free $\mathrm{ddH}_{2} \mathrm{O}$ up to $20 \mu \mathrm{L}$. The condition for reverse

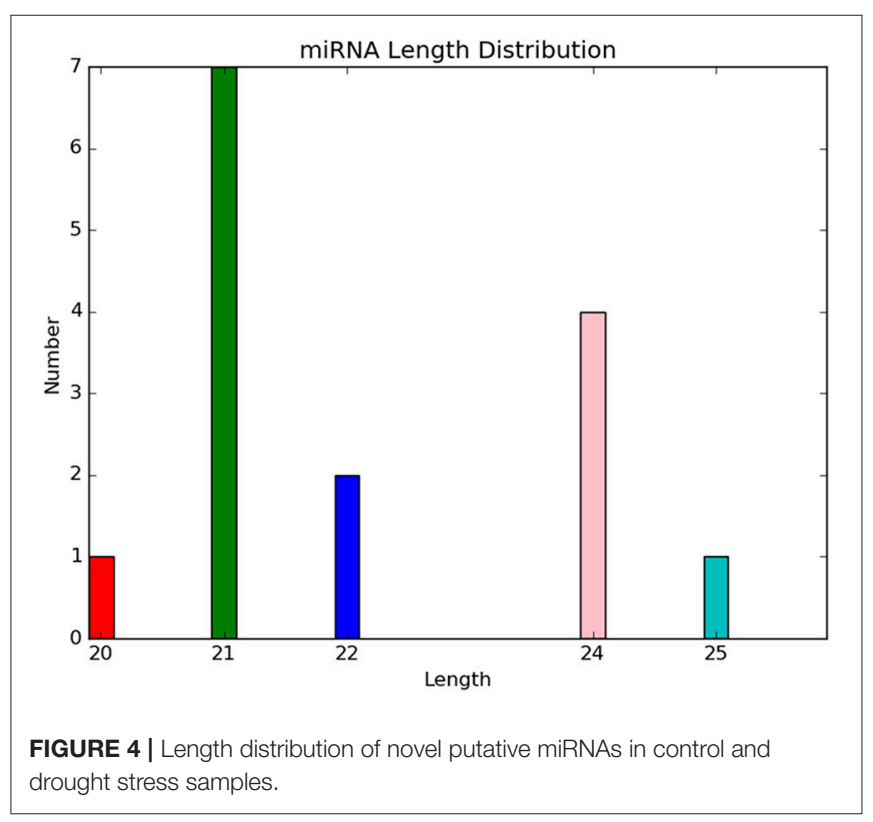

transcription reaction was set at $37^{\circ} \mathrm{C}$ for $15 \mathrm{~min}$ followed by $85^{\circ} \mathrm{C}$ for $1 \mathrm{~min}$.

RT-qPCR of miRNAs and their target genes was performed on an ABI 7,500 Real-Time PCR system using SYBR Premix Ex $\mathrm{Taq}^{\mathrm{TM}}$ and SYBR Green (TaKaRa, Japan) following the manufacturer's instructions. PCR reactions of miRNAs were performed at $95^{\circ} \mathrm{C}$ for $10 \mathrm{~min}$, followed by 40 cycles of $95^{\circ} \mathrm{C}$ for $20 \mathrm{~s}, 60^{\circ} \mathrm{C}$ for $30 \mathrm{~s}$, and a cycle of dissociation at $95^{\circ} \mathrm{C}$ for $15 \mathrm{~s}$, $60^{\circ} \mathrm{C}$ for $30 \mathrm{~s}, 95^{\circ} \mathrm{C}$ for $15 \mathrm{~s}$. PCR reactions of target genes were performed as follows: $95^{\circ} \mathrm{C} 1 \mathrm{~min} ; 40$ cycles of $95^{\circ} \mathrm{C} 10 \mathrm{~s}, 60^{\circ} \mathrm{C}$ $30 \mathrm{~s}$, and $72^{\circ} \mathrm{C} 30 \mathrm{~s}$. The primers used are listed in Table 1. U6 (a kind of snRNA that forms ribonucleoprotein) and wheat unigene Ta54825 (Actin) were used as internal control to analyze the relative expression level of miRNAs and target genes, respectively. Calculation of relative expression levels of genes was done by the $\Delta \Delta^{\mathrm{Ct}}$ method (Pfaffl, 2001). The expression levels of genes are presented as means calculated from three replicates. 

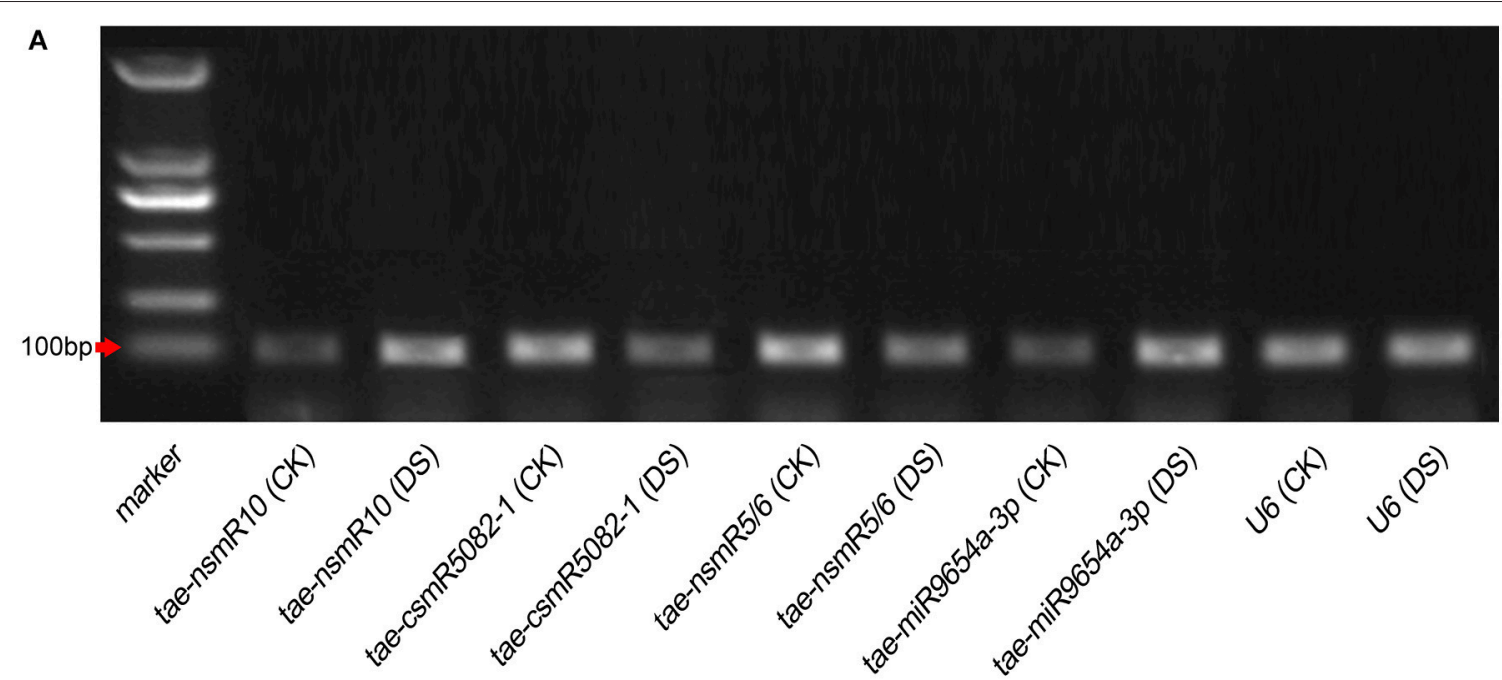

B

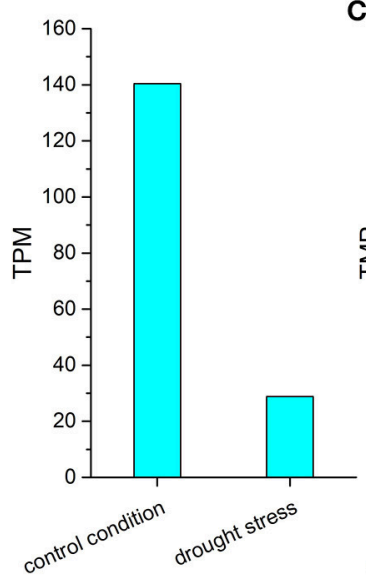

C

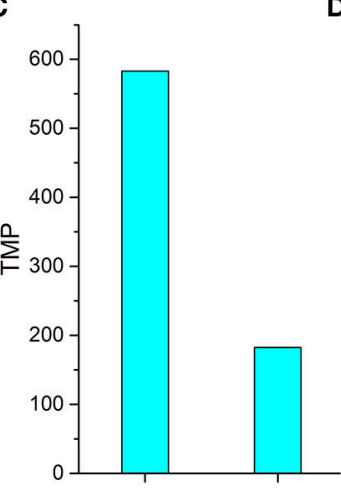

control con arition aroughts stress

$\mathbf{F}$

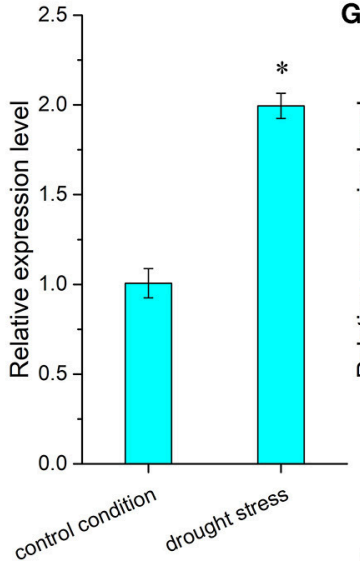

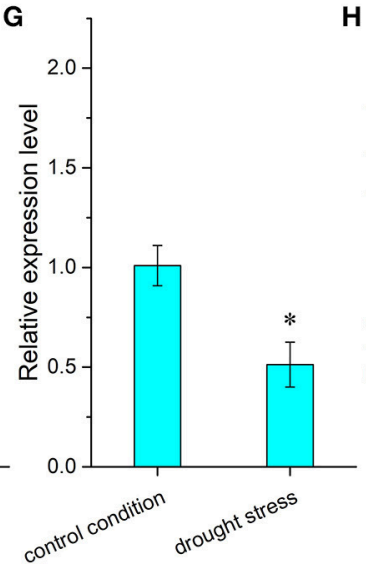

D

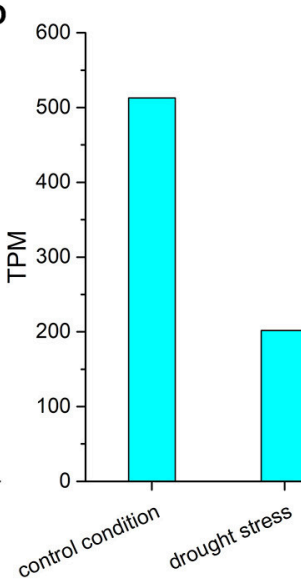

E
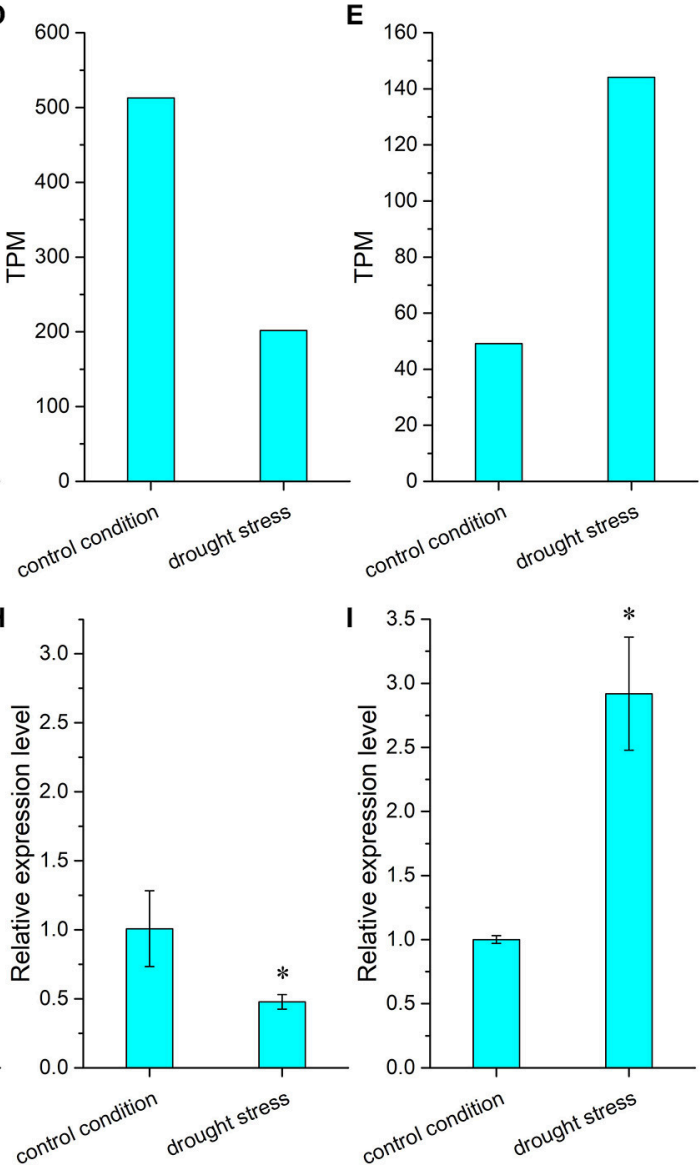

control condition droughts trese

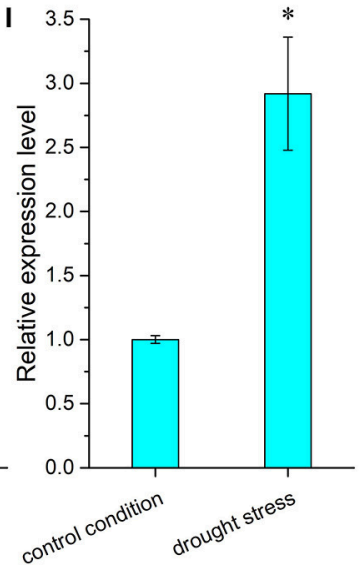

FIGURE 5 | Differential expression analysis of miRNA in caryopses under drought stress. (A) Expression profile of differentially expressed miRNA by RT-PCR. CK indicates control condition and DS indicates drought stress. (B,F) TPM value and relative expression level of tae-nsmR10; (C,G) TPM value and relative expression level of tae-csmR5082-1; (D,H) TPM value and relative expression level of tae-nsmR5/6; (E,I) TPM value and relative expression level of tae-miR9654a-3p. Each miRNA expression value was normalized against the internal control gene U6. Relative expression levels were presented as mean \pm SE, and means were calculated from three replicates. Asterisks between the control and drought stress indicate significant difference at $p<0.05$ as determined by $t$-test.

\section{Statistical Analysis}

Data analyses were conducted using Excel 2016 (Microsoft, USA). T-test was used to compare the means at a significance level of $p<0.05$. Origin 8.0 (OriginLab, USA) and Photoshop CS5 (Adobe, USA) were used to illustrate the figures. 
TABLE 4 | Prediction and functional annotation of differentially expressed miRNA targets.

\begin{tabular}{lll}
\hline miRNA ID & Targets ID & Annotation \\
\hline tae-csmR5082-1 & TC390380 & Cationic peroxidase SPC4 \\
& CA637513 & Arabinogalactan peptide \\
& CA644040 & Arabinogalactan peptide \\
& CA634690 & Arabinogalactan peptide \\
& TC436117 & Arabinogalactan peptide \\
& CA645741 & IAA-amino acid hydrolase \\
& TC389427 & Predicted protein \\
& TC438668 & Hypothetical protein \\
& TC449449 & Hypothetical protein \\
& CA610228 & Peroxidase \\
& CA663463 & RUBISCO activase beta \\
& TC395269 & beta-1,3-galactosyltransferase \\
& TC432653 & DEAD-box ATP-dependent RNA helicase \\
& TC369499 & 1-aminocyclopropane-1-carboxylate oxidase \\
\hline tae-nsmR5, tae-nsmR6 & TC435511 & 3-mercaptopyruvate sulfurtransferase \\
& TC371915 & 3-mercaptopyruvate sulfurtransferase \\
\hline tae-nsmR10 & TC436629 & Proline-rich receptor \\
CA678419 & Peroxiredoxin-2C \\
DR735664 & SNF1-related protein kinase catalytic subunit \\
TC458111 & Hypothetical protein \\
CA646062 & Peroxiredoxin-2C \\
CJ793000 & Trehalose-phosphate phosphatase \\
& TC422325 & Sulfated surface glycoprotein \\
DR735126 & Shikimate dehydrogenase \\
DR733425 & Vesicle-associated protein \\
BJ258028 & Predicted protein \\
CJ698196 & Predicted protein \\
TC426820 & Heterogeneous nuclear ribonucleoprotein \\
\hline & & \\
\hline
\end{tabular}

\section{RESULTS}

\section{Sequence Analysis of Small RNAs}

After sequencing, 15,536,948 and 10,340,923 effective reads were obtained from wheat caryopses under control and drought stress, respectively. Most of effective reads were annotated into different classes and mapped to the reference genomes (Table 2). As a result, the total tags and unique tags mapped to the reference genomes accounted for the largest proportion, followed by the percentage of total tags and unique tags annotated as rRNA. The rRNA amount can be used as a quality control standard for samples, and the rRNA proportion in plant RNA samples is generally $<60 \%$. Up to $38.22 \%$ of the total tags and $8.07 \%$ of the unique tags were annotated as rRNA in the control group. Moreover, the proportion of total tags and unique tags annotated as rRNA in the drought-treated group were 42.62 and $9.71 \%$, respectively. This indicated that our sequencing data were of good quality. Up to 7,272,595 (46.81\%) total tags and 3,664,651 (75.67\%) unique tags in the control group were mapped to the reference genomes. Meanwhile, in the treatment group, the total tags and unique tags mapped to the reference genome were $4,574,247$ (44.23\%) and 2,549,296 (74.43\%), respectively. These effective reads mapped to the reference genomes were used for subsequent miRNA identification.

The length distribution of small RNAs and common and specific sequence analysis of total tags and unique tags between control and drought stress are shown in Figure 2. Under control and drought conditions, the length distributions of the total tags and unique tags were basically similar but uneven. The number of total tags and unique tags with $24 \mathrm{nt}$ in length was the largest, and the rest were mainly distributed between 19 and 23 nt (Figures 2A,B). These results are similar to the length distribution of small RNAs in plants. Furthermore, 6,982,596 (36.95\%) total tags and 740,677 (9.84\%) unique tags were the common sequences between the two samples (Figure 2C). This indicated good consistency of the samples in sequencing, and that the sequencing results were indeed of good representativeness.

\section{Identification, Length Distribution Statistics, and Base Preference Analysis of miRNAs}

By aligning with known miRNAs in miRBase and predicting novel miRNAs, 125 miRNAs were identified in the control and drought stress groups. This included 110 known miRNAs and 15 novel putative miRNAs [miRNA specific sequence information is shown in Supplementary Material 1 (Table)]. Specific expression of tae-miR399 was found in the control sample, whereas taemiR5085, tae-miR5200, and tae-miR6197-5p were specifically expressed in the drought sample. Length distribution of all identified miRNAs showed that the majority of miRNA lengths were in the range of 20-24 nt (Figure 3A). Considering that dicer enzymes specifically recognize and cleave precursor miRNAs, the first base site of mature miRNA displayed strong preference to U. Moreover, the other sites displayed a certain base bias, which can be used to evaluate the sequencing accuracy. The base distribution analysis of miRNA at each site showed that the content of $\mathrm{C}$ was low, and the contents of $\mathrm{G}, \mathrm{U}$, and $\mathrm{A}$ were similar (Figure 3B).

Five novel putative miRNAs were classified as conservative miRNAs, and 10 were unconservative miRNAs. Their specific sequence information is shown in Table 3. Length distribution analysis showed that the novel miRNAs with a length of 20 nt were the most followed by $24 \mathrm{nt}$ miRNAs (Figure 4). The novel putative miRNA precursor structure was predicted using the miRDeep2 software. Detailed information is shown in Supplementary Material 2 (Figure).

\section{Differential Expression Analysis of miRNA}

Expression analysis of miRNA under drought stress is shown in Figure 5. Differences in miRNA expression profiles were investigated by RT-PCR (Figure 5A). The transcript signals of miRNA tae-nsmR10 and tae-miR9654a-3p in caryopses under drought stress were higher than those in control caryopses. However, transcript accumulations of miRNA tae-csmR5082-1 and tae-nsmR5/6 were decreased under drought stress. The TPM value and relative expression levels of differentially expressed miRNAs in control and drought samples were calculated. Based on high-throughput sequencing data and bioinformatic 


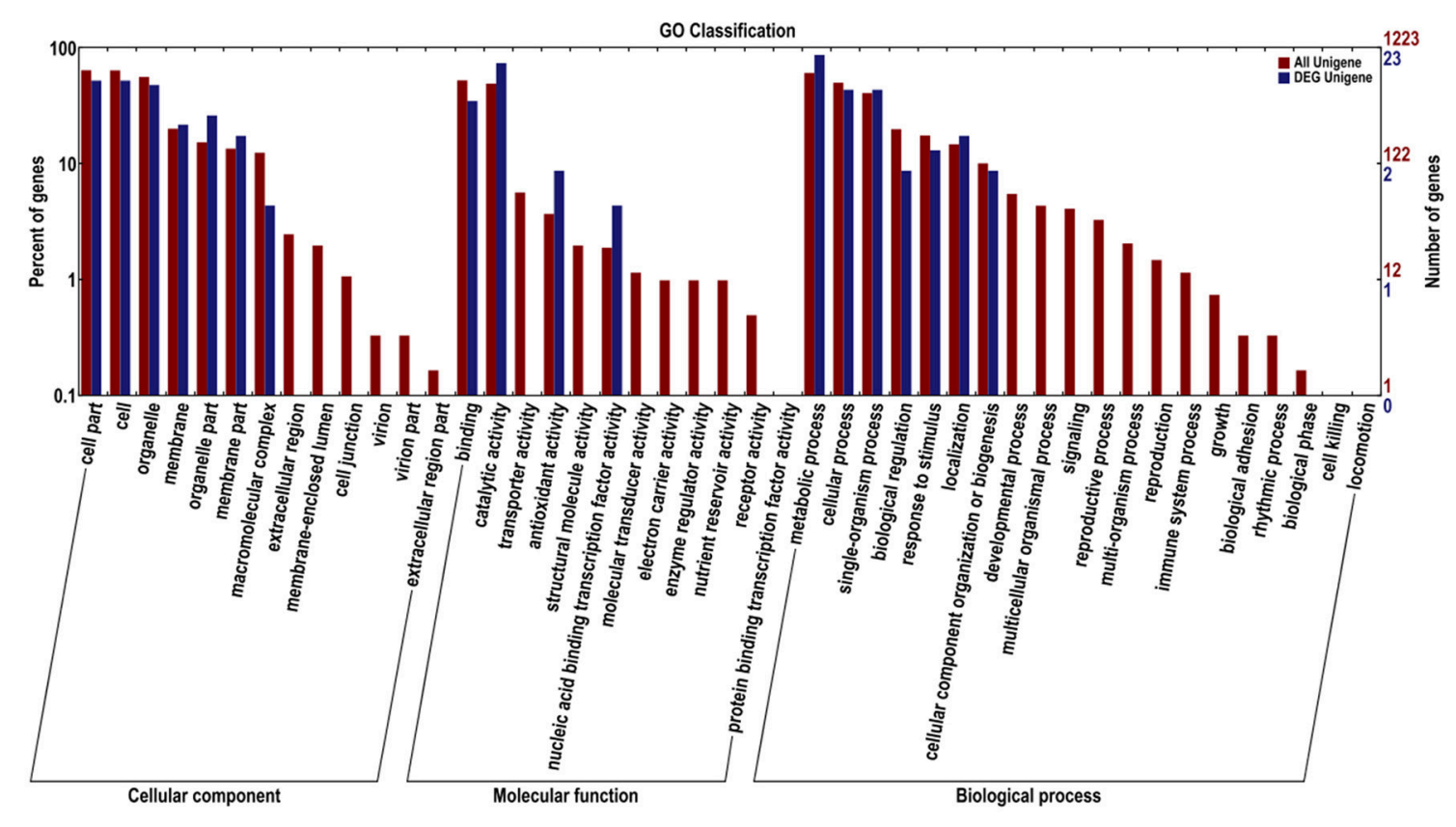

FIGURE 6 | GO enrichment functional analysis of miRNA targets. Red represents target genes for all miRNAs, and blue represents target genes for differentially expressed miRNAs.

analysis, four miRNAs with significantly differential expression were singled out, namely, tae-nsmR10, tae-csmR5082-1, taensmR5/tae-nsmR6 (Tae-nsmR5 and tae-nsmR6 were considered to be the same miRNA because they had the same sequence) and tae-miR9654a-3p. The expression of known miRNA taemiR9654a-3p was up-regulated, whereas the other three novel miRNAs were down-regulated after drought stress treatment (Figures 5B-E). To further validate the expression profile of differentially expressed miRNA under drought stress, the relative expression levels of the above four miRNAs were analyzed by RT-qPCR. The relative expression level of taensmR10 and tae-miR9654a-3p exhibited the up-regulated pattern while that of tae-csmR5082-1 and tae-nsmR5/taensmR6 exhibited the down-regulated pattern under drought stress (Figures 5F-I). In addition, the expression trend of three miRNAs (tae-csmR5082-1, tae-nsmR5/tae-nsmR6 and taemiR9654a-3p) showed consistent results between the TPM value and relative expression level determined by high-throughput sequencing and RT-qPCR, respectively (Figures 5C,G,D,H,E,I). However, for tae-nsmR10, the TPM value was inconsistent with the relative expression level (Figures 5B,F).

\section{Prediction and Functional Analysis of Differentially Expressed miRNA Target Genes}

Target genes of all identified miRNAs were predicted using psRNATarget software and compared with the relevant database for functional annotation. For all the identified miRNAs, 1,981 target genes were predicted. Of these, 1,641 target genes were annotated. Interestingly, each miRNA can have different targets, and different miRNAs can have the same target gene. For differentially expressed miRNAs, 28 predicted target genes were annotated with function and specific information as shown in Table 4.

Functional annotation based on GO and KEGG databases revealed that miRNA targets had various functions, involving biological processes, molecular genetics and other aspects. Moreover, GO enrichment analysis of all identified miRNAs and differentially expressed miRNA targets was performed and the results are shown in Figure 6. According to GO functional annotation, the target genes of miRNA were classified into three categories, namely, biological process, cellular component, and molecular function. For the cellular component category, numerous target genes for all identified and differentially expressed miRNAs fell under cell part, cell, organelle, membrane, organelle part, membrane component, and macromolecular complex subcategories. Moreover, the extracellular region comprised the smallest proportion of all miRNA targets. In the molecular functional category, the two most abundant subcategories for all miRNAs were binding and catalytic activity. The target genes of differentially expressed miRNAs were classified under binding, catalytic activity, antioxidant activity, and nucleic acid binding transcription factor activity. Under the biological process category, majority of all miRNA targets and all the differentially expressed miRNA targets were enriched under the following secondary function entries: metabolic processes, cellular process, single-organism process, biological regulation, response to stimulus, localization, and cellular component organization or biogenesis. Moreover, the enrichment trends of all miRNA targets and differentially expressed miRNA targets in GO entries were similar. 


\section{A}

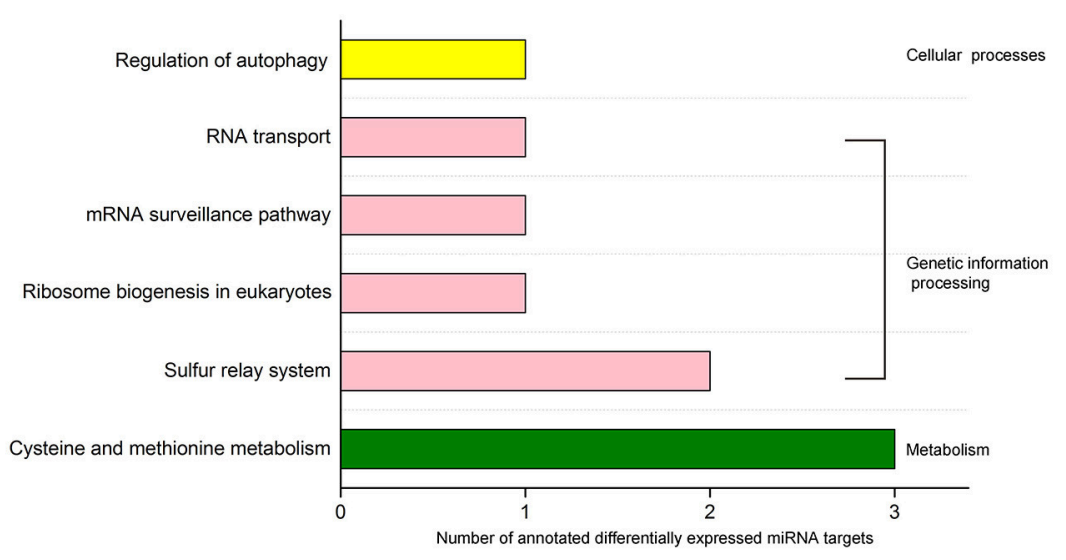

B

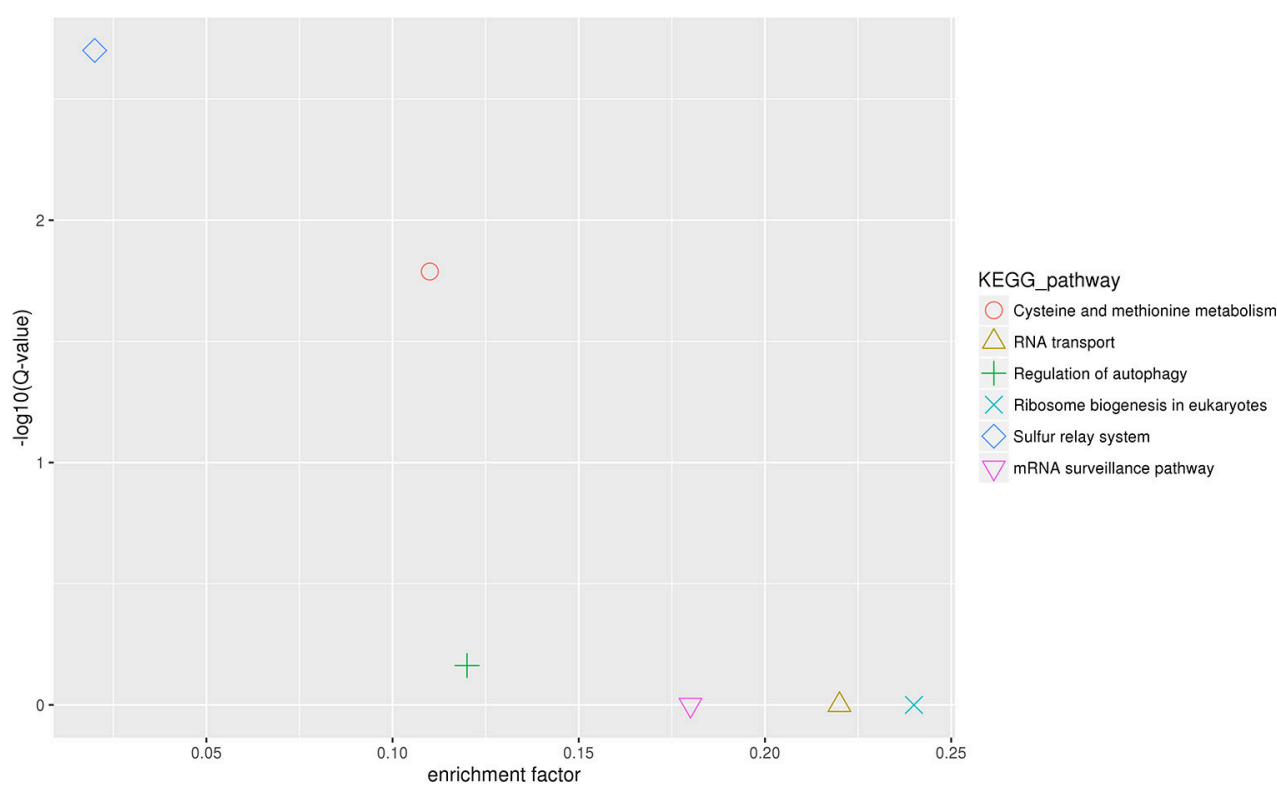

FIGURE 7 | KEGG pathway analysis of differentially expressed miRNA targets. (A) KEGG classification of differentially expressed miRNA targets. (B) KEGG pathway enrichment scatter diagram of target genes of differentially expressed miRNAs.

Classification of the KEGG pathway and enrichment analysis of the pathway were performed based on KEGG annotation of differentially expressed miRNA targets (Figure 7). The differentially expressed miRNA target genes were annotated into the following six KEGG pathways: cysteine and methionine metabolism, sulfur relay system, RNA transport, ribosome biogenesis in eukaryotes, mRNA surveillance pathway, and autophagy regulation. These six pathways can be classified into three categories, namely, metabolism, genetic information processing, and cellular processes. The pathway with the largest number of annotated target genes was cysteine and methionine metabolism, followed by sulfur relay system (Figure 7A). KEGG pathway enrichment analysis showed that the two pathways with the highest enrichment significance were sulfur relay system and cysteine and methionine metabolism (Figure 7B). We screened four KEGG pathways that may be involved in protein synthesis and found differentially expressed miRNA target gene-related enzymes from the pathway maps: 3mercaptopyruvate sulfurtransferase (MPST) in cysteine and methionine metabolism, and aminocyclopropane carboxylate oxidase, RNA-binding protein Musashi in mRNA surveillance pathway, and nonsense-mediated mRNA decay protein 3 in ribosome biogenesis in eukaryotes and RNA transport.

\section{RT-qPCR Analysis of miRNA Target Genes}

To further understand the expression patterns of miRNA target genes, we implemented RT-qPCR to determine gene relative expression levels. We selected one corresponding target gene for each differentially expressed miRNA. The selected genes were TC436629, TC369499, TC371915, and DR733425, which encode proline-rich receptor, 1-aminocyclopropane-1carboxylate oxidase, MPST and vesicle-associated protein, respectively (bold values in Table 4). The relative expression levels of these genes are shown in Figure 8. Drought stress 


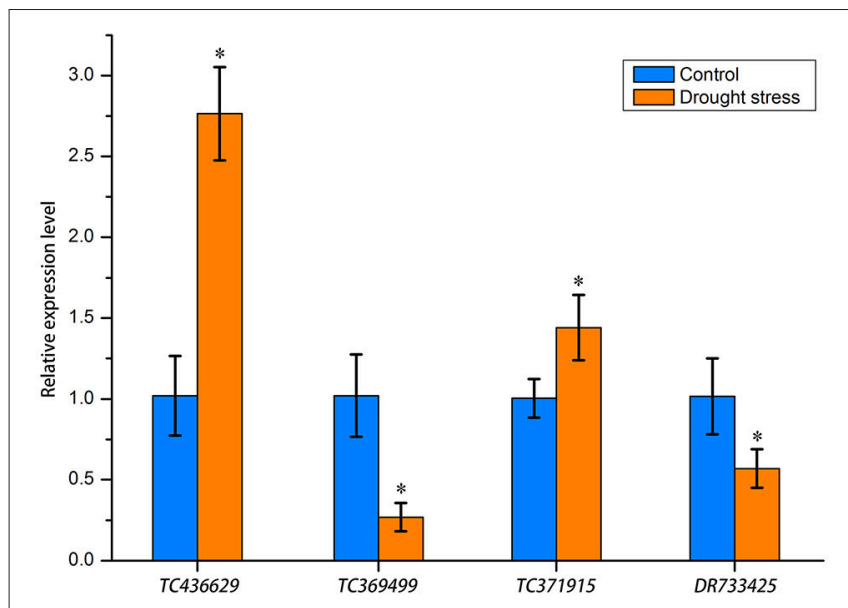

FIGURE 8 | Relative expression levels of four miRNA target genes determined by RT-qPCR. Gene expression levels were normalized against the internal control gene Ta54825. Asterisks between the control and drought stress indicate significant difference at $p<0.05$ as determined by $t$-test. Means were calculated from three replicates.

increased the relative expression levels of TC436629 and TC371915 and decreased the relative expression levels of TC369499 and DR733425. Compared with the expression tendency of differentially expressed miRNA in Figure 5, three miRNA-target gene pairs showed opposite trends, defined as negative miRNA regulation. These miRNAs were tae-nsmR10, tae-nsmR5/tae-nsmR6, and tae-miR9654a-3p.

\section{Accumulation of Protein Bodies and Protein Content in Wheat Endosperm under Drought Stress}

The accumulation characteristics of protein bodies in wheat endosperm under drought stress were observed using the semi-thin slice technique. Starch and proteins stored in the amyloid and $\mathrm{PB}$, respectively, are the main reserve substances in wheat endosperm. At $15 \mathrm{DAA}$, there were no remarkable changes in caryopsis profiles (Figure 9A) and endosperm cells were substantially enriched by starch granules and protein bodies. The protein bodies mainly accumulated in the vicinity of the aleurone layer (Figures 9B,D). Some small protein bodies were observed in endosperm cells under the control condition, whereas large protein aggregations were observed in the endosperm cells of drought-stressed wheat (Figures 9C,E). These findings indicated that drought stress increased the accumulation of protein bodies in the endosperm. Furthermore, we determined the protein content in wheat caryopsis at 15 and 45 DAA (Figure 9F). The results showed that protein contents were significantly enhanced by drought stress at these two stages.

\section{DISCUSSION}

Drought stress is one of the important abiotic stress factors that affects wheat yield and grain quality. To cultivate high-yielding crop varieties with improved stress resistance, the complex molecular mechanisms underlying stress response must be thoroughly understood. Several studies have reported that miRNA plays a significant role in plant response to stress ( $\mathrm{Lu}$ and Huang, 2008; Eldem et al., 2012). Therefore, identifying and manipulating some abiotic stress-related miRNA regulatory molecules may facilitate improvement in stress resistance and grain yield in cereals (Hikmet et al., 2015), such as wheat (Qu et al., 2015). To date, the methods for studying miRNA are mainly bioinformatics-based prediction and experimentation. Different research methods can be selected for different research purposes.

In recent years, high-throughput sequencing of small RNA has been used to analyze the specific and differential expression of miRNA in plants at different developmental stages, in different tissues, or under different stress treatments. In previous researches, numerous miRNAs were determined in crop species under different stress conditions like barley (Schreiber et al., 2011; Hackenberg et al., 2015) and rice (Yi et al., 2013). Moreover, Ren (2011) identified 21 down-regulated miRNAs and nine up-regulated miRNAs under cold stress in poplar by using the high-throughput sequencing technique. Jian et al. (2010) conducted high-throughput sequencing of five small RNA libraries under drought stress, salt stress, cold stress, and abscisic acid stress, together with a control group. Numerous specifically and differentially expressed miRNAs were found under different stress factors in rice. These studies about miRNA identification based on high-throughput sequencing provide a platform for further analysis of expression profiles of miRNAs and characterization of stress-responsive miRNAs in plants. However, only a few drought responsive miRNAs are known among the miRNAs identified in wheat. Of the 125 miRNAs identified in the present study, only four (tae-nsmR10, tae-csmR50821, tae-nsmR5/tae-nsmR6, tae-miR9654a-3p) were found to be differentially expressed under drought stress. Similar results were found in barley which reported that four of 28 miRNAs identified in barley were differentially expressed under dehydration stress conditions (Kantar et al., 2010). The regulation of differentially expressed miRNAs induced by drought may be partly associated with drought-related transcription factors (TFs) such as dehydration-responsive element binding TFs (Morran et al., 2011).

Identifying target genes that are regulated by stress-responsive miRNAs is important for revealing possible roles of miRNA in regulating wheat response to abiotic stress. Based on the high sequence complementarity of mature plant miRNAs and corresponding targets, target genes can be predicted using bioinformatic tools ( $\mathrm{Ku}$ et al., 2015). Using psRNATarget and BLAST software to predict and annotate putative miRNA targets at the genome level has become one of the most popular approaches (Pandey et al., 2014; Ma et al., 2015). However, the putative target genes need to be further validated by further experiments such as degradome sequencing, which is an effective tool to identify target genes directly cleaved by miRNA on a large scale (German et al., 2008). In the present study, 1,981 target genes were predicted, of which 1,641 target genes were annotated. Quantitative PCR results can reveal the relationship between miRNAs and target genes in stress-responsive expressions. 

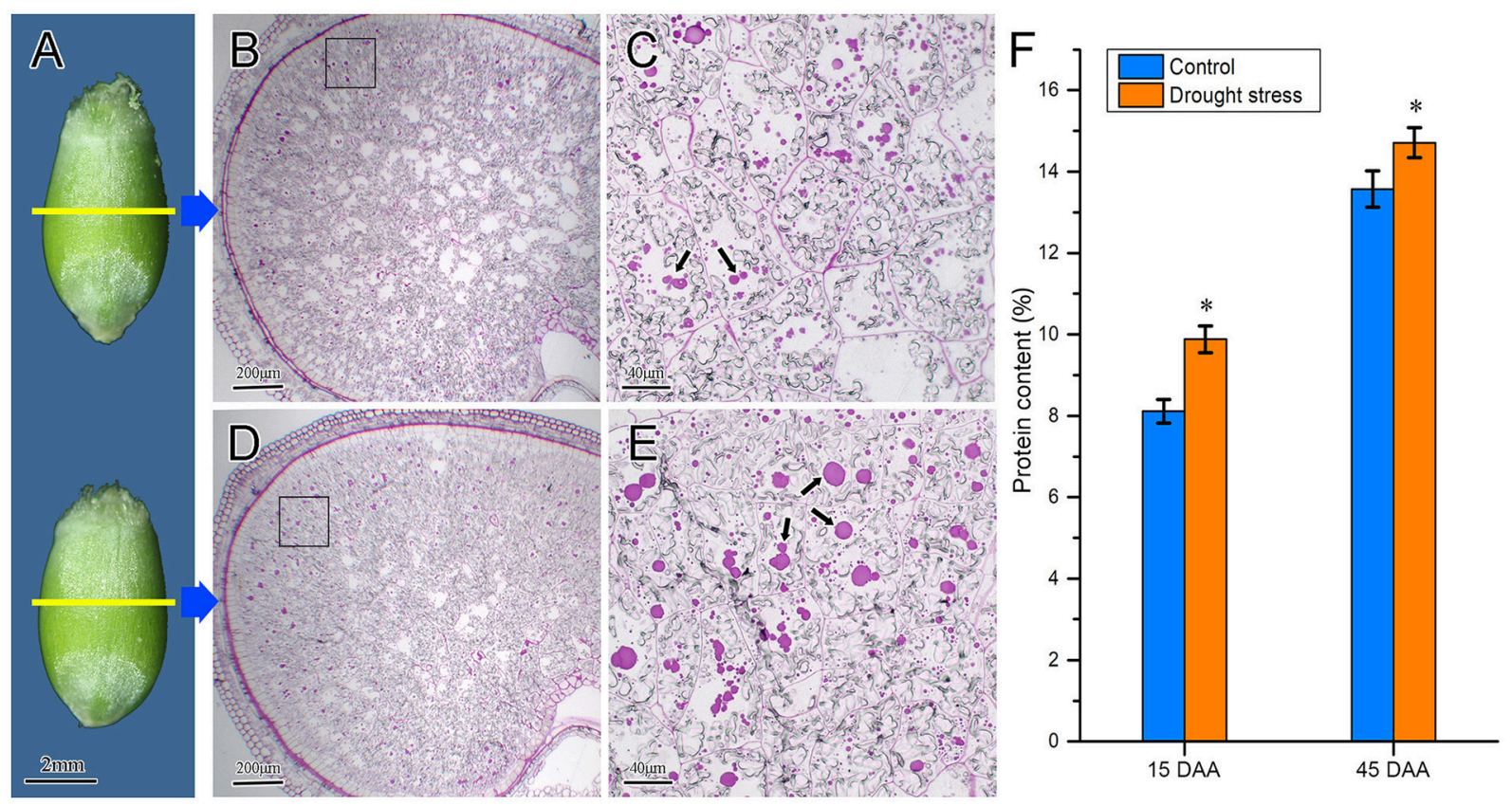

FIGURE 9 | Difference of protein body accumulation in wheat endosperm under control and drought stress conditions. (A) Wheat caryopsis profile. The caryopses at the top and the bottom represent control and drought stress conditions, respectively. (B,C) Microstructure of wheat endosperm under control condition; (D,E) microstructure of wheat endosperm under drought stress. Subpanels (C,E) are high-magnification views of the black boxes in subpanels (B,D), respectively. Black arrows in the figures indicate protein bodies. (F) Protein contents of wheat caryopsis at 15 and 45 DAA under drought stress. Data are expressed as means from three individual biological replicates. Asterisks above the histogram indicate a significant difference at $p<0.05$ as determined by $t$-test. Scale bars: (A) 2 mm, (B,D) $200 \mu \mathrm{m}$, and $(\mathbf{C}, \mathbf{E}) 40 \mu \mathrm{m}$.

The interaction between miRNA and target genes is complex based on their expression profiles. When a miRNA targets multiple genes, the miRNA only exhibits negative correlation with a certain target gene (Liang et al., 2014). In this study, four differentially expressed miRNAs were identified, and these miRNAs corresponded to 28 target genes. Using RT-qPCR, we analyzed four corresponding target genes of four differentially expressed miRNAs. The results showed that all of them presented different expression profiles. Additionally, three miRNA-target gene pairs (tae-nsmR10-TC436629, tae-nsmR5/tae-nsmR6TC371915 and tae-miR9654a-3p-DR733425) exhibited opposite expression tendencies. This opposite expression tendency has been previously reported (Tang et al., 2014; Wen et al., 2016) and considered as negative regulation of miRNA (Kumar et al., 2015). However, one miRNA-target pair (tae-csmR5082-1-TC369499) exhibited similar expression trend, which was inconsistent with the negative regulation theory of miRNA. This may be because the specific miRNA silencing of a certain target gene under drought stress occurs only at a certain development stage or tissue, whereas other target genes remain unaffected.

It is revealed that drought stress can alter expression of many genes and metabolites, including dehydrins, vacuolar acid invertase, glutathione S-transferase (GST), late embryo abundant (LEA), RuBisCo, helicase, proline, and carbohydrates (Nezhadahmadi et al., 2013). As the regulator of gene expression, miRNA is deemed to participate in the regulation of these drought responsive genes. In our study, 28 target genes of four differentially expressed miRNAs were annotated with a wide range of biological functions. Of these, four target genes (TC390380, CA610228, CA678419, CA646062) encoded the peroxidase (POD) component. Antioxidant enzymes like POD have the function on elimination of reactive oxygen species and alleviation of drought injury. Wei et al. (2009) reported that down-regulated miR168 and miR528 under drought resulted in up-regulated expression of the target gene mitogen-activated protein kinase (MAPK), which further induced the expression of antioxidant genes and antioxidant enzymes. In addition, the target genes CA663463 and TC436629 encoded rubisco activase beta and proline-rich receptor, respectively, both of which were related to drought resistance.

In general, miRNA interacts with Argonaute protein to form RNA-induced silencing complexes and specifically bind to the mRNA of target gene by directly cleaving target mRNA or inhibiting protein translation (Carrington and Ambros, 2003), thereby resulting in negative regulation of gene expression at transcriptional and post-transcriptional levels (Guo et al., 2010). The interactions between miRNAs and their target genes through sequence-specific binding provide an accurate regulation pathway for plants to respond to environmental stimuli. Based on KEGG pathway analysis, differentially expressed miRNA targets were found to be enriched in six pathways. Several target genes, which encoded 3-mercaptopyruvate sulfurtransferase (MPST) and aminocyclopropane carboxylate oxidase (ACO), were enriched in cysteine and methionine metabolism pathway, which is an important route for synthesis of sulfur-containing amino acids (Goyer et al., 2007). ACO is known to be involved 
in ethylene biosynthesis which is related to leaf senescence in drought adaptation (Gómez-Lim et al., 1993). Moreover, nonsense-mediated mRNA decay protein 3 (NMD3) in RNA transport and ribosome biogenesis pathway is also the expression product of differentially expressed miRNA targets, which is key to nuclear export of ribosomal subunits (Chen et al., 2012).

Since the aforementioned pathways were both related to protein biosynthesis, one interpretation of our results is that protein biosynthesis in caryopsis may be regulated by protein synthesis-relevant KEGG pathways. However, the expression level of target genes in these pathways under drought needs further validation. According to gene function analysis, the target of miR9654a-3p is related to vesicle transport of protein in cells. Meanwhile, expression analysis indicated that the up-regulated expression trend of miR9654a-3p was opposite to that of its target gene, which exhibited a down-regulated expression pattern under drought stress. This is predicted to cause decrease in protein output and increase in protein accumulation. These results showed that miR9654a-3p can regulate the expression of coatomer protein-related genes and affect vesicle transport of protein in cells. This may explain previously documented increase in $\mathrm{PB}$ accumulation in the endosperm under drought stress. Thus, we concluded that drought stress may affect development of protein bodies in endosperm through the regulation of miRNAs involved in protein synthesis and vesicle transport.

\section{CONCLUSION}

The present study identified 125 miRNAs in wheat caryopses under control and drought stress conditions, including 110 known miRNAs and 15 putative novel miRNAs. Four

\section{REFERENCES}

Ambros, V. (2004). The functions of animal microRNAs. Nature 431, 350-355. doi: 10.1038 /nature 02871

Bartel, D. P. (2004). MicroRNAs: genomics, biogenesis, mechanism, and function. Cell 116, 281-297. doi: 10.1016/S0092-8674(04)00045-5

Buermans, H. P., Ariyurek, Y., Ommen, G. V., Dunnen, J. T. D., and Hoen, P. A. T. (2010). New methods for next generation sequencing based microRNA expression profiling. BMC Genomics 11:716. doi: 10.1186/1471-216411-716

Carrington, J. C., and Ambros, V. (2003). Role of microRNAs in plant and animal development. Science 301, 336-338. doi: 10.1126/science.10 85242

Chaves, M. M., Maroco, J. P., and Pereira, J. S. (2003). Understanding plant responses to drought - from genes to the whole plant. Funct. Plant Biol. 30, 239-264. doi: 10.1071/FP02076

Chen, G., Zhu, J., Zhou, J., Subburaj, S., Ming, Z., Han, C., et al. (2014). Dynamic development of starch granules and the regulation of starch biosynthesis in Brachypodium distachyon: comparison with common wheat and Aegilops peregrina. BMC Plant Biol. 14:198. doi: 10.1186/s12870-0140198-2

Chen, M. Q., Zhang, A. H., Zhang, Q., Zhang, B. C., Nan, J., Li, X., et al. (2012). Arabidopsis NMD3 is required for nuclear export of 60S ribosomal subunits and affects secondary cell wall thickening. PLoS ONE 7:e35904. doi: 10.1371/journal.pone.0035904 differentially expressed miRNAs induced by drought stress may affect the development of protein bodies in caryopsis by regulating the expression levels of target genes involved in protein biosynthesis pathways. These findings offered a putative mechanism underlying the increased accumulation of storage proteins in wheat endosperm under drought stress.

\section{AUTHOR CONTRIBUTIONS}

$\mathrm{XC}$ analyzed the data and wrote the paper. YY performed resin slicing and drew the figures. LR accomplished the RNA extraction and RT-qPCR analysis. ZD collected the experimental samples and materials. EZ determined the protein content. XY supervised the experiments. FX designed the experiments and critically revised the paper.

\section{ACKNOWLEDGMENTS}

This study was supported by the Natural Science Foundation of China (31571573, 31701351), the Natural Science Foundation of Jiangsu Province (Grant No. BK20170497), the Natural Science Foundation of the Jiangsu Higher Education Institutions of China (Grant No. 17KJD180007), a Project Funded by the Priority Academic Program Development of Jiangsu Higher Education Institutions (PAPD), the Foundation of Excellent Doctoral Dissertation of Yangzhou University.

\section{SUPPLEMENTARY MATERIAL}

The Supplementary Material for this article can be found online at: http://journal.frontiersin.org/article/10.3389/fpls.2017. 01707/full\#supplementary-material
Chen, X. (2005). MicroRNA biogenesis and function in plants. FEBS Lett. 579, 5923-5931. doi: 10.1016/j.febslet.2005.07.071

Consortium, T. G. O., Ashburner, M., Ball, C. A., Blake, J. A., Botstein, D., Butler, H., et al. (2000). Gene ontology: tool for the unification of biology. Nat. Genet. 25, 25-29. doi: 10.1038/75556

Covarrubias, A. A., and Reyes, J. L. (2009). Post-transcriptional gene regulation of salinity and drought responses by plant microRNAs. Plant Cell Environ. 33, 481-489. doi: 10.1111/j.1365-3040.2009.02048.x

Drea, S., Leader, D. J., Arnold, B. C., Shaw, P., Dolan, L., and Doonan, J. H. (2005). Systematic spatial analysis of gene expression during wheat caryopsis development. Plant Cell 17, 2172-2185. doi: 10.1105/tpc.105. 034058

Eldem, V., Celikkol Akcay, U., Ozhuner, E., Bakir, Y., Uranbey, S., and Unver, T. (2012). Genome-wide identification of miRNAs responsive to drought in peach (Prunus persica) by high-throughput deep sequencing. PLoS ONE 7:e50298. doi: 10.1371/journal.pone.0050298

Enright, A. J., John, B., Gaul, U., Tuschl, T., Sander, C., and Marks, D. S. (2003). MicroRNA targets in Drosophila. Genome Biol. 5:R1. doi: 10.1186/gb-2003-5-1-r1

Friedländer, M. R., Mackowiak, S. D., Li, N., Chen, W., and Rajewsky, N. (2012). miRDeep2 accurately identifies known and hundreds of novel microRNA genes in seven animal clades. Nucleic Acids Res. 40, 37-52. doi: 10.1093/nar/ gkr688

German, M. A., Pillay, M., Jeong, D. H., Hetawal, A., Luo, S. J., Janardhanan, P., et al. (2008). Global identification of microRNA-target RNA pairs by 
parallel analysis of RNA ends. Nat. Biotechnol. 26, 941-946. doi: 10.1038/ nbt1417

Gómez-Lim, M. A., Valdés-López, V., Cruz-Hernandez, A., and SaucedoArias, L. J. (1993). Isolation and characterization of a gene involved in ethylene biosynthesis from Arabidopsis thaliana. Gene 134, 217-221. doi: 10.1016/0378-1119(93)90096-L

Goyer, A., Collakova, E., Shachar-Hill, Y., and Hanson, A. D. (2007). Functional characterization of a methionine $\gamma$-lyase in Arabidopsis and its implication in an alternative to the reverse trans-sulfuration pathway. Plant Cell Physiol. 48, 232-242. doi: 10.1093/pcp/pcl055

Guo, H., Ingolia, N. T., Weissman, J. S., and Bartel, D. P. (2010). Mammalian microRNAs predominantly act to decrease target mRNA levels. Nature 466, 835-840. doi: 10.1038/nature09267

Habash, D. Z., Kehel, Z., and Nachit, M. (2009). Genomic approaches for designing durum wheat ready for climate change with a focus on drought. J. Exp. Bot. 60, 2805-2815. doi: 10.1093/jxb/erp211

Hackenberg, M., Gustafson, P., Langridge, P., and Shi, B. J. (2015). Differential expression of microRNAs and other small RNAs in barley between water and drought conditions. Plant Biotechnol. J. 13, 2-13. doi: 10.1111/pbi.12220

Hikmet, B., Melda, K., Reyyan, B., and Bala Ani, A. (2015). Stress responsive miRNAs and isomiRs in cereals. Plant Sci. 235, 1-13. doi: $10.1016 /$ j.plantsci.2015.02.008

Jagadeeswaran, G., Saini, A., and Sunkar, R. (2009). Biotic and abiotic stress down-regulate miR398 expression in Arabidopsis. Planta 229, 1009-1014. doi: 10.1007/s00425-009-0889-3

Jian, X. Y., Lin, Z., Li, G. L., Liang, Z., Wang, X. J., Cao, X. F., et al. (2010). Identification of novel stress-regulated microRNAs from Oryza sativa L. Genomics 95, 47-55. doi: 10.1016/j.ygeno.2009.08.017

Kanehisa, M., Goto, S., Kawashima, S., Okuno, Y., and Hattori, M. (2004). The KEGG resource for deciphering the genome. Nucleic Acids Res. 32, D277-D280. doi: 10.1093/nar/gkh063

Kantar, M., Unver, T., and Budak, H. (2010). Regulation of barley miRNAs upon dehydration stress correlated with target gene expression. Funct. Integr. Genomics 10, 493-507. doi: 10.1007/s10142-010-0181-4

Kindred, D. R., Verhoeven, T. M. O., Weightman, R. M., Swanston, J. S., Aguc, R. C., Brosnan, J. M., et al. (2008). Effects of variety and fertiliser nitrogen on alcohol yield, grain yield, starch and protein content, and protein composition of winter wheat. J. Cereal Sci. 48, 46-57. doi: 10.1016/j.jcs.2007.07.010

Ku, Y. S., Wong, J. W., Mui, Z., Liu, X., Hui, J. H., Chan, T. F., et al. (2015). Small RNAs in plant responses to abiotic stresses: regulatory roles and study methods. Int. J. Mol. Sci. 16, 24532-24554. doi: 10.3390/ijms161024532

Kumar, R. R., Pathak, H., Sharma, S. K., Kala, Y. K., Nirjal, M. K., Singh, G. P., et al. (2015). Novel and conserved heat-responsive microRNAs in wheat (Triticum aestivum L.). Funct. Integr. Genomics 15, 323-348. doi: 10.1007/s10142-014-0421-0

Langmead, B. (2010). Aligning short sequencing reads with Bowtie. Curr Protoc. Bioinform. Chapter 11(Unit 11.17), Unit 11.17. doi: 10.1002/0471250953.bi1107s32

Lawlor, D. W. (2013). Genetic engineering to improve plant performance under drought: physiological evaluation of achievements, limitations, and possibilities. J. Exp. Bot. 64, 83-108. doi: 10.1093/jxb/ers326

Li, T., Ma, L., Geng, Y. K., Hao, C. Y., Chen, X. H., and Zhang, X. Y. (2015). Small RNA and degradome sequencing reveal complex roles of miRNAs and their targets in developing wheat grains. PLoS ONE 10:e139658. doi: 10.1371/journal.pone.0139658

Liang, G., He, H., Li, Y., Wang, F., and Yu, D. (2014). Molecular mechanism of microRNA396 mediating pistil development in arabidopsis. Plant Physiol. 164, 249-258. doi: 10.1104/pp.113.225144

Liu, F. L., Jensen, C. R., and Andersen, M. N. (2005). A review of drought adaptation in crop plants: changes in vegetative and reproductive physiology induced by ABA-based chemical signals. Aust. J. Agric. Res. 56, 1245-1252. doi: 10.1071/AR05062

Lu, X. Y., and Huang, X. L. (2008). Plant miRNAs and abiotic stress responses. Biochem. Biophys. Res. Commun. 368, 458-462. doi: 10.1016/j.bbrc.2008.02.007

Ma, X., Xin, Z., Wang, Z., Yang, Q., Guo, S., Guo, X., et al. (2015). Identification and comparative analysis of differentially expressed miRNAs in leaves of two wheat (Triticum aestivum L.) genotypes during dehydration stress. BMC Plant Biol. 15:21. doi: 10.1186/s12870-015-0413-9
Mallory, A. C., and Bouché, N. (2008). MicroRNA-directed regulation: to cleave or not to cleave. Trends Plant Sci. 13, 359-367. doi: 10.1016/j.tplants.2008.03.007

Morran, S., Eini, O., Pyvovarenko, T., Parent, B., Singh, R., Ismagul, A., et al. (2011). Improvement of stress tolerance of wheat and barley by modulation of expression of DREB/CBF factors. Plant Biotechnol. J. 9, 230-249. doi: 10.1111/j.1467-7652.2010.00547.x

Nezhadahmadi, A., Prodhan, Z. H., and Faruq, G. (2013). Drought tolerance in wheat. Sci. World J. 2013:610721. doi: 10.1155/2013/610721

Pandey, R., Joshi, G., Bhardwaj, A. R., Agarwal, M., and Katiyaragarwal, S. (2014). A comprehensive genome-wide study on tissue-specific and abiotic stress-specific miRNAs in Triticum aestivum. PLoS ONE 9:e95800. doi: 10.1371/journal.pone.0095800

Pfaffl, M. W. (2001). A new mathematical model for relative quantification in real-time RT-PCR. Nucleic Acids Res. 29:e45. doi: 10.1093/nar/29.9.e45

Qu, B., He, X., Wang, J., Zhao, Y., Teng, W., Shao, A., et al. (2015). A wheat CCAAT box-binding transcription factor increases the grain yield of wheat with less fertilizer input. Plant Physiol. 167, 411-423. doi: 10.1104/pp.114. 246959

Ren, Y. (2011). Genome-wide identification of cold-responsive and new microRNAs in Populus tomentosa by high-throughput sequencing. Biochem. Biophys. Res. Commun. 417, 892-896. doi: 10.1016/j.bbrc.2011.12.070

Reyes, J. L., and Chua, N. H. (2007). ABA induction of miR159 controls transcript levels of two MYB factors during Arabidopsis seed germination. Plant J. 49, 592-606. doi: 10.1111/j.1365-313X.2006.02980.x

Rhoades, M. W., Reinhart, B. J., Lim, L. P., Burge, C. B., Bartel, B., and Bartel, D. P. (2002). Prediction of plant microRNA targets. Cell 110, 513-520. doi: 10.1016/S0092-8674(02)00863-2

Romualdi, C., Bortoluzzi, S., D’Alessi, F., and Danieli, G. A. (2003). IDEG6: a web tool for detection of differentially expressed genes in multiple tag sampling experiments. Physiol. Genomics 12, 159-162. doi: 10.1152/physiolgenomics.00096.2002

Schreiber, A. W., Shi, B. J., Huang, C. Y., Langridge, P., and Baumann, U. (2011). Discovery of barley miRNAs through deep sequencing of short reads. BMC Genomics 12:129. doi: 10.1186/1471-2164-12-129

Shinozaki, K., and Yamaguchi-Shinozaki, K. (2007). Gene networks involved in drought stress response and tolerance. J. Exp. Bot. 58, 221-227. doi: $10.1093 /$ jxb/erl164

Song, J. B., Huang, S. Q., Dalmay, T., and Yang, Z. M. (2012). Regulation of leaf morphology by microRNA394 and its target LEAF CURLING RESPONSIVENESS. Plant Cell Physiol. 53, 1283-1294. doi: $10.1093 / \mathrm{pcp} / \mathrm{pcs} 080$

Tang, M., Mao, D., Xu, L., Li, D., Song, S., and Chen, C. (2014). Integrated analysis of miRNA and mRNA expression profiles in response to $\mathrm{Cd}$ exposure in rice seedlings. BMC Genomics 15:835. doi: 10.1186/1471-216415-835

Tasleem-Tahir, A., Nadaud, I., Chambon, C., and Branlard, G. (2012). Expression profiling of starchy endosperm metabolic proteins at 21 stages of wheat grain development. J. Proteome Res. 11, 2754-2773. doi: 10.1021/pr201110d

Verdier, J., and Thompson, R. D. (2008). Transcriptional regulation of storage protein synthesis during dicotyledon seed filling. Plant Cell Physiol. 49, 1263-1271. doi: 10.1093/pcp/pcn116

Wei, L., Zhang, D. F., Xiang, F., and Zhang, Z. X. (2009). Differentially expressed miRNAs potentially involved in the regulation of defense mechanism to drought stress in maize seedlings. Int. J. Plant Sci. 170, 979-989. doi: $10.1086 / 605122$

Wen, M., Xie, M., He, L., Wang, Y., Shi, S., and Tang, T. (2016). Expression variations of miRNAs and mRNAs in rice (Oryza sativa). Genome Biol. Evol. 8, 3529-3544. doi: 10.1093/gbe/evw252

Windels, D., and Vazquez, F. (2011). miR393: integrator of environmental cues in auxin signaling? Plant Signal. Behav. 6, 1672-1675. doi: 10.4161/psb.6.11. 17900

Yi, R., Zhu, Z., Hu, J., Qian, Q., Dai, J., and Ding, Y. (2013). Identification and expression analysis of microRNAs at the grain filling stage in rice(Oryza sativa L.) via deep sequencing. PLoS ONE 8:e57863. doi: 10.1371/journal.pone.0057863

Zhang, N., Yang, J., Wang, Z., Wen, Y., Wang, J., He, W., et al. (2014). Identification of novel and conserved microRNAs related to drought stress in potato by deep sequencing. PLoS ONE 9:e95489. doi: 10.1371/journal.pone.0095489 
Zhao, B., Liang, R., Ge, L., Li, W., Xiao, H., Lin, H., et al. (2007). Identification of drought-induced microRNAs in rice. Biochem. Biophys. Res. Commun. 354, 585-590. doi: 10.1016/j.bbrc.2007.01.022

Zhou, L., Chen, J., Li, Z., Li, X., Hu, X., Huang, Y., et al. (2010). Integrated profiling of microRNAs and mRNAs: microRNAs located on Xq27.3 associate with clear cell renal cell carcinoma. PLoS ONE 5:e15224. doi: 10.1371/journal.pone.0015224

Zhou, M., Gu, L. F., Li, P. C., Song, X. W., Wei, L. Y., Chen, Z. Y., et al. (2010). Degradome sequencing reveals endogenous small RNA targets in rice (Oryza sativa L. ssp. indica). Front. Biol. 5, 67-90. doi: 10.1007/s11515-010$0007-8$
Conflict of Interest Statement: The authors declare that the research was conducted in the absence of any commercial or financial relationships that could be construed as a potential conflict of interest

Copyright (c) 2017 Chen, Yang, Ran, Dong, Zhang, Yu and Xiong. This is an openaccess article distributed under the terms of the Creative Commons Attribution License (CC BY). The use, distribution or reproduction in other forums is permitted, provided the original author(s) or licensor are credited and that the original publication in this journal is cited, in accordance with accepted academic practice. No use, distribution or reproduction is permitted which does not comply with these terms. 